\title{
"NOSSO MAIS VALIOSO RECURSO": MENSURANDO O IMPACTO DOS PROGRAMAS DE INTERCÂMBIO ESTADUNIDENSES SOBRE O COMPORTAMENTO INTERNACIONAL DE OUTROS ESTADOS
}

\begin{abstract}
Resumo
O artigo testa a hipótese de que países tendem a apresentar um comportamento internacional mais favorável em relação aos EUA quando seus chefes de Estado tiveram contato prévio com instituições de ensino estadunidenses. Foi feito um levantamento prosopográfico dos chefes de Estado dos países que compõem o Grupo Latino-Americano e Caribenho na Organização das Nações Unidas (GRULAC-ONU), observando especificamente quais destes tiveram contato com instituições de ensino estadunidenses. Para medir o posicionamento em relação aos EUA, foi adotada como variável proxy os padrões de votação desses Estados na Assembleia Geral da ONU, comparados aos padrões de votação estadunidenses, recorrendo a base de dados compilada por Voeten, Strezhnev e Bailey (2009). O marco inicial escolhido para a pesquisa foi o fim da Guerra Fria (dezembro de 1991), se estendendo até dezembro de 2017. Por se tratarem de duas variáveis dicotômicas, foi calculado o Coeficiente de Associação de Yule para verificar a força da relação entre elas. Todas as variações do teste retornaram uma associação positiva baixa, flutuando entre 0,108 (analisando todas as votações e considerando contato com universidades estadunidenses ou de outros membros da OTAN) a 0,202 (analisando apenas as votações consideradas importantes pelos EUA e considerando apenas o contato com universidades estadunidenses). Os resultados forneceram evidências que corroboram com a hipótese de que o contato prévio com instituições de ensino estadunidenses por parte de chefes de Estado produz uma postura mais positiva dessas nações em relação aos EUA
\end{abstract}

Palavras-Chave: Comportamento internacional; Assembleia Geral da ONU; Socialização de chefes de Estado; Intercambios Educacionais.

\section{INTRODUÇÃO}

Não é nova a ideia de que os intercâmbios acadêmicos em instituições de ensino norteamericanas são um instrumento relevante para ampliar a influência dos Estados Unidos pelo mundo. Sua lógica fundamental gira em torno da ideia de socializar os valores e a cultura norte-americana em jovens que, talvez um dia, farão parte das elites governantes de seus respectivos países. Conforme expresso em um relatório de um grupo educacional norte-americano, "as milhões de pessoas que têm estudado nos Estados Unidos ao longo dos anos constituem um notável reservatório de boa vontade

\footnotetext{
${ }^{1}$ Doutorando pelo Programa de Pós-Graduação em Ciência Política da Universidade Federal do Paraná (PRPPG-UFPR). E-mail: douglashnovelli@ outlook.com. ORCID: https://orcid.org/0000-0001-6058-5109.
} 
para o nosso país" (NAFSA, 2003, tradução nossa) ${ }^{2}$; de modo semelhante, Colin Powell (2001, tradução nossa) ${ }^{3}$, então Secretário de Estado dos Estados Unidos, em 2001 declarou que ele não era capaz de pensar em nenhum recurso mais valioso para os EUA do que "a amizade dos futuros líderes mundiais aqui educados"; enquanto Joseph Nye (2004, p. 45, tradução nossa) ${ }^{4}$ aponta que intercâmbios acadêmicos e científicos "desempenham um papel significativo em aumentar o soft power norte-americano".

Até certo ponto, essas estratégias vêm sendo comprovadas por pesquisas empíricas, que ao longo dos anos têm consistentemente evidenciado como estudantes de intercâmbio tendem a retornar para seus países de origem com uma visão mais positiva do país no qual estudaram (Richmond, 2003; Selltiz et al., 1963; Wang, 1991; Watson e Lippitt, 1958; Wilson e Bonilla, 1955). Contudo, a produção de resultados políticos a nível internacional, derivados desses intercambios, apesar de muito especulada, ainda carece de estudos empíricos que a sustente. A presente pesquisa se volta para essa lacuna, explorando o argumento de que Estados são mais propensos a adotar um comportamento favorável aos interesses estadunidenses quando seus respectivos líderes vivenciaram períodos de socialização em instituições de ensino estadunidenses.

Para tanto, o presente artigo adota uma estrutura de cinco seções em seu desenvolvimento, iniciando com uma breve revisão teórica sobre o conceito de socialização, seguida de uma seção voltada para a apresentação dos argumentos e construção das hipóteses que serão testadas, exposição dos materiais e métodos empregados, apresentação dos resultados e, por fim, a discussão e as considerações finais, nas quais são sugeridos tópicos para uma agenda de pesquisa futura.

\section{REVISÃO TEÓRICA}

Desde o início do século XXI, autores como Alastair Johnston (2001) e Jeffrey Checkel (2005) vêm apontando que o conceito de socialização tem sido particularmente subutilizado no campo das Relações Internacionais, especialmente se comparado às demais ciências humanas, nas quais frequentemente exerce posição de destaque ${ }^{5}$. Do ponto de vista teórico, mesmo se forem

\footnotetext{
2 "The millions of people who have studied in the United States over the years constitute a remarkable reservoir of goodwill for our country."

3 "I can think of no more valuable asset to our country than the friendship of future world leaders who have been educated here."

4 "Academic and scientific exchanges played a significant role in enhancing American soft power."

${ }^{5}$ Exemplos incluem a Sociologia e a Psicologia Social, com teorias sobre formação de identidade social e cumprimento das normas do grupo (Cialdini, 1987; Napier e Gershenfeld, 1987; Nisbett e Cohen, 1996; Turner, 1987); a Ciência
} 
consideradas as vertentes teóricas das RI que lhe dão alguma significância, como o Construtivismo e a Escola Inglesa, em geral o conceito de socialização ainda parece ser subteorizado e ter seus microprocessos majoritariamente ignorados. Já no que toca aos estudos empíricos, o conceito tem sido principalmente empregado por trabalhos voltados a entender como o ambiente internacional pode influenciar positivamente nos processos domésticos de democratização, seja através do papel socializador exercido pelas organizações internacionais sobre as elites políticas domésticas (Bellin, 2012; Dimitrova e Pridham, 2004; Greenhill, 2010; Kelley, 2004; Pevehouse, 2002a; b, 2005); ou através do impacto causado pelo intercâmbio de oficiais estrangeiros em academias militares estadunidenses sobre o desenvolvimento de instituições democráticas em seus países de origem (Atkinson, 2006, 2010; Cope, 1995; Miller, 2006; Ruby e Gibler, 2010).

Conforme apontado por Beyers (2010), socialização diz respeito ao processo pelo qual indivíduos desenvolvem uma ligação com um grupo social e adaptam seu comportamento para se adequar as expectativas advindas do mesmo. Nas palavras de Stryker e Statham (1985, p. 325, tradução nossa) ${ }^{6}$, "socialização é o termo genérico utilizado para designar os processos pelos quais o recém-chegado - seja ele o recém-nascido, o novato ou o estagiário, por exemplo - se incorpora nos padrões organizados de interação". Sua função básica é criar um senso de pertencimento social, pelo qual os entendimentos intersubjetivos dessa sociedade são internalizados e tomados como fatos (Johnston, 2001). Implica que o agente socializado evolua de uma lógica de consequências para uma lógica de adequação, com suas ações sendo tomadas independentemente de qualquer estrutura de incentivos ou sanções em particular, sendo a aceitação das normas e expectativas socialmente impostas internalizadas como "a coisa certa a ser feita” (Checkel, 2005, p. 804, tradução nossa) ${ }^{7}$.

O mecanismo causal da socialização, tal qual instrumentalizado pelos estudos que tratam da socialização democrática promovida por intercâmbios acadêmicos, envolve a alteração de atitudes em relação a determinado tema ou agente, promovido graças a experiências pessoais em redes políticas criadas e controladas em torno desses temas ou por esses agentes. Assim, um conceito acessório imprescindível ao de socialização é o conceito de atitudes, que podem ser entendidas como “disposições avaliativas” (Cooper, Blackman e Keller, 2016; Freyburg, 2011), que são aprendidas e podem ser alteradas através da comunicação social ou de experiências pessoais diretas, podendo

Política, com estudos sobre a formação de orientações políticas entre jovens e explicações para movimentos sociais (Beck e Jennings, 1991); e o Direito Internacional, com pesquisas sobre o papel da condenação social para assegurar o cumprimento de tratados (Chayes e Chayes, 1996; Moravcsik, 1995; Susskind, 1994; Young, 1992).

6 "Socialization is the generic term used to refer to the processes by which the newcomer - the infant, rookie, or trainee, for example - becomes incorporated into organized patterns of interaction."

7 " the right thing to do". 
envolver tanto componentes afetivos (baseados em emoções) quanto cognitivos (baseados em crenças). Nas palavras de Perloff (2017, p. 89-90, tradução nossa $)^{8}$ :

\begin{abstract}
Ter uma atitude significa que você classificou algo e fez um julgamento do seu valor. Significa que você já não é neutro em relação ao tema. Isso não significa que não possa ter sentimentos mistos, mas a sua opinião sobre o assunto já não é neutra ou isenta de cor. [...] Atitudes (e valores) organizam o nosso mundo social. Elas nos permitem categorizar pessoas, lugares e eventos rapidamente e descobrir o que está acontecendo. São como marca páginas, etiquetas para categorizar uma coleção de livros favoritos, ou formas de organizar aplicativos para smartphones. As atitudes moldam as percepções e influenciam os julgamentos. Se você é republicano, provavelmente avalia os líderes políticos republicanos favoravelmente e tem uma reação negativa, ao nível do instinto, a alguns políticos democratas. E vice-versa, se for um democrata. Por outro lado, se odeia a política e desconfia dos políticos, filtra o mundo político através de um conjunto de lentes céticas.
\end{abstract}

Assim, conforme resumido por Lomer (2017), o argumento central, empregado por trabalhos que analisam o potencial socializador de intercâmbios acadêmicos, gira em torno da concepção de que estudantes internacionais tendem a alterar suas atitudes e se identificar com o país receptor como um resultado de experiências positivas no processo de socialização. O intercâmbio acadêmico em instituições de ensino superior seria particularmente efetivo ao promover a socialização desses estudantes pois, conforme apontam Gift e Krcmaric (2017, p. 5, tradução nossa) ${ }^{9}$, “o ensino superior [...] está entre os meios mais importantes através dos quais os indivíduos desenvolvem crenças políticas", sendo "o início da idade adulta [...] o período mais formativo da vida de uma pessoa". Ademais, como apontam autores como Anna Wojciuka, Maciej Michałekb e Marta Stormowskac (2015), atualmente a educação parece ser "um valor universal”, com aspectos como sua qualidade e alcance sendo apreciados independentemente da cultura e do país, ao passo que é individualmente valorizada como condição para a prosperidade econômica - tornando-se assim um veículo ideal para "ganhar corações e mentes" (Nye, 2008). Destarte, tal qual intercâmbios acadêmicos apresentam o potencial para estimular a socialização democrática ao alterar as atitudes dos participantes em relação a democracia graças a promoção de experiências pessoais em redes políticas criadas e controladas

\footnotetext{
8 "Having an attitude means that you have categorized something and made a judgment of its net value or worth. It means that you are no longer neutral about the topic. That doesn't mean you can't have mixed feelings, but your view on the issue is no longer bland or without color. [...] Attitudes (and values) organize our social world. They allow us to categorize people, places, and events quickly and to figure out what's going on. They are like notebook dividers, labels to categorize a collection of favorite books, or ways to organize smartphone apps. Attitudes shape perceptions and influence judgments. If you're a Republican, you probably evaluate Republican political leaders favorably and have a negative, gut-level reaction to some Democratic politicians. And vice versa if you are a Democrat. On the other hand, if you hate politics and distrust politicians, you filter the political world through a skeptical set of lenses."

9 "Higher education is an especially powerful form of interpersonal contact because it is among the most important ways in which individuals develop political beliefs and because early adulthood is arguably the most formative period of a person's life".
} 
por democracias estabelecidas (Freyburg, 2011), é possível que esse mecanismo também possa gerar nos participantes atitudes positivas duradouras em relação aos Estados receptores, que podem vir a se converter em ganhos políticos para esses Estados caso esses estudantes um dia ocupem posições políticas decisórias em seus países de origem. O presente artigo explora essa ideia, focando especificamente em chefes de Estado que tiveram contato com instituições de ensino norteamericanas.

\section{CONSTRUÇÃO DE HIPÓTESES TESTÁVEIS}

$\mathrm{O}$ artigo testa o argumento base de que um Estado é mais propenso a adotar um comportamento internacional favorável aos EUA quando seu atual chefe de Estado teve contato prévio com instituições de ensino estadunidenses. Isto posto, definir parâmetros para o que pode ser considerado um comportamento internacional favorável aos EUA é uma problemática de difícil resolução, tendo em vista a natureza multidimensional das relações internacionais. Contudo, a literatura tem demonstrado que existem fortes evidências de que o governo norte-americano atribui importância real aos resultados das votações na Assembleia geral das Nações Unidas (AGNU), exercendo pressão política sobre seus pares com o objetivo de assegurar resultados que lhe sejam favoráveis (ver: Andersen, Harr e Tarp, 2006; Bennis, 1997; Dreher, Nunnenkamp e Thiele, 2008; Thacker, 1999). Conforme exposto por Bailey, Strezhnev e Voeten (2015, p. 2, tradução nossa) ${ }^{10}$, “as votações na Assembleia Geral das Nações Unidas (AGNU) tornaram-se a fonte de dados normalizada para a construção de medidas de preferências estatais, uma vez que são ações comparáveis e observáveis tomadas pelos países em determinados momentos”. Assim, os padrões de votação na AGNU podem ser efetivamente aplicados como uma variável proxy para medir o comportamento internacional de outros Estados em relação aos EUA, pois, ainda que não contemplem totalmente a complexidade das relações que podem ser estabelecidas entre estes Estados, esses padrões de voto fornecem sólidos indícios sobre o posicionamento geral de seus atores.

Baseado nesses argumentos, derivamos a primeira hipótese da pesquisa:

\footnotetext{
10 "Votes in the United Nations General Assembly (UNGA) have become the standard data source for constructing measures of state preferences, as they are comparable and observable actions taken by many countries at set points in time."
} 


\section{$H_{1}$ : Um Estado é mais propenso a votar junto com EUA na AGNU quando seu chefe de Estado} teve contato prévio com instituições de ensino norte-americanas.

Um fator relevante que precisa ser levado em consideração é a ampla variedade de pautas que se tornam objeto de votações na AGNU. Como apontam Dreher, Nunnenkamp e Thiele (2008), é provável que nem todos os votos sejam de importância para o governo norte-americano, de forma que restringir a análise apenas as votações que abordem pautas importantes pode acabar gerando resultados mais significativos. Embora rotular as votações como importantes ou não seja um processo altamente subjetivo, esse desafio pode ser solucionado ao utilizar a própria categorização empregada pelo Departamento de Estado norte-americano, que desde 1983 indica quais foram as "votações sobre questões que afetaram diretamente interesses importantes dos Estados Unidos e em relação às quais os Estados Unidos fizeram um amplo lobby" (US Public Law 101-246, apud United States Department of State, 2018, tradução nossa) ${ }^{11}$. Assim, a segunda hipótese deriva-se de uma variação direta da primeira, limitando a análise exclusivamente aos votos codificados como importantes pelo governo dos EUA.

$\mathrm{H}_{2}$ : Um Estado é mais propenso a votar junto com EUA na AGNU, em pautas consideradas importantes pelo governo norte-americano, quando seu chefe de Estado teve contato prévio com instituições de ensino norte-americanas.

Ao trabalhar com os votos da AGNU, ainda é relevante considerar os padrões históricos de aliança que neles podem ser observados. Ao longo das décadas, pesquisadores tem demonstrado como os Estados do ocidente mantém um padrão de votos coeso e próximo entre si, sobretudo nos casos nos quais existem alianças formais entre esses atores, como a União Europeia e a Organização do Tratado do Atlântico Norte (OTAN) (Campbell, 1971; Holloway, 2009; Kim e Russett, 1996; Potrafke, 2009). Assim, é razoável supor a possibilidade de que países governados por chefes de Estado que tenham passado por um período de socialização acadêmica em Estados aliados aos EUA, aqui recortados como os membros da OTAN, também apresentem um padrão de votos mais alinhado aos interesses norte-americanos. Dessa ideia, derivam as hipóteses finais que serão testadas:

\footnotetext{
11 "votes on issues which directly affected important United States interests and on which the United States lobbied extensively"
} 
H3: Um Estado é mais propenso a votar junto com EUA na AGNU quando seu chefe de Estado teve contato prévio com instituições de ensino norte-americanas, ou de outros membros da OTAN.

H4: Um Estado é mais propenso a votar junto com EUA na AGNU, em pautas consideradas importantes pelo governo norte-americano, quando seu chefe de Estado teve contato prévio com instituições de ensino norte-americanas, ou de outros membros da OTAN.

\section{MATERIAIS E MÉTODOS}

\subsection{MÉTODOS DE COLETA E DESCRIÇÃO DOS DADOS}

A pesquisa recorreu a duas bases de dados distintas, derivando suas conclusões a partir da confrontação de ambas.

Em primeiro lugar, para medir o posicionamento de cada um dos Estados analisados em relação aos EUA, foi adotada, como variável proxy, os padrões de votação desses Estados na Assembleia Geral das Nações Unidas (AGNU), comparados aos padrões de votação estadunidenses. Para tanto, foi utilizada a base de dados já compilada por Bailey, Strezhnev e Voeten (2009), como parte do projeto Harvard Dataverse ${ }^{12}$.

De início, foi necessário aplicar dois recortes, um com relação aos Estados que seriam observados e outro com relação ao recorte temporal que seria considerado pela pesquisa. No que toca ao primeiro recorte, foi feita a opção pelos Estados que compõem o Grupo Latino-Americano e Caribenho na Organização das Nações Unidas (GRULAC-ONU) ${ }^{13}$, escolha que se deu tendo em vista a proximidade geográfica com os Estados Unidos. Ao menos em teoria, essa proximidade amplia as chances de localizar políticos que tenham tido contato prévio com instituições de ensino norteamericanas antes de assumirem os cargos de chefe de Estado em seus respectivos países, possibilitando assim que a análise seja feita com base em um número razoável de notações heterogêneas. Já no que toca ao recorte temporal da pesquisa, optou-se por iniciar a análise imediatamente após o fim da Guerra Fria, em dezembro de 1991, se estendendo até o ponto no qual os dados haviam sido compilados, em dezembro de 2017.

\footnotetext{
${ }^{12}$ Disponível em: <http://dx.doi.org/10.7910/DVN/LEJUQZ〉.

${ }^{13}$ Os Estados membros do GRULAC são: Antígua e Barbuda, Argentina, Bahamas, Barbados, Belize, Bolívia, Brasil, Chile, Colômbia, Costa Rica, Cuba, Dominica, Republica Dominicana, Equador, El Salvador, Granada, Guatemala, Guiana, Haiti, Honduras, Jamaica, México, Nicarágua, Panamá, Paraguai, Peru, Santa Lúcia, São Cristóvão e Nevis, São Vicente e Granadinas, Suriname, Trinidad e Tobago, Uruguai e Venezuela.
} 
Ao todo, existem quatro possibilidades de voto que os Estados podem tomar em cada votação: sim; não; abstenção; e ausência. Seguindo o padrão utilizado por autores como Kegley e Hook $(1991)^{14}$, foram descartadas ocorrências nas quais os EUA, ou o Estado ao qual sua votação era comparada, se abstiveram ou estavam ausentes. Do mesmo modo, seguindo Dreher e Sturm (2006), foram excluídos os casos de votação por consenso, nos quais todos os Estados analisados votaram da mesma forma. Os votos dos EUA foram então individualmente comparados com aqueles feitos pelos Estados do GRULAC, notando como 1 os votos iguais (entre os EUA e determinado Estado em dada votação) e 0 para os votos destoantes. Seguindo essas regras de recorte e tratamento, foram totalizadas 32.461 notações individuais ao longo de todo o corpus, coletadas em 1.180 votações.

Por sua vez, a segunda base de dados foi compilada especificamente para a execução da presente pesquisa. A base em questão foi desenvolvida através de um levantamento prosopográfico dos chefes de Estado que estiveram à frente dos países que compõem o GRULAC no período analisado, observando especificamente quais destes tiveram contato com instituições de ensino superior ou militares estadunidenses, seja na qualidade de discentes ou como docentes. Ao todo, foram coletados dados de 189 políticos, dos quais 62 (32,8\%) haviam tido contato com instituições de ensino norte-americanas e 39 (20,6\%) haviam tido contato com instituições de ensino de outros Estados membros da OTAN ${ }^{15}$.

\subsection{MÉTODOS DE ANÁLISE}

Por se tratarem de duas variáveis dicotômicas, foi calculado o Coeficiente de Associação de Yule $\left(Q_{x y}\right)$ para verificar a força da relação entre elas. Conforme exposto por Cervi (2019, p. 36), “o teste de independência $Q_{x y}$ serve para identificar se: i) duas variáveis dicotômicas estão relacionadas entre si; ii) de quanto é a intensidade da relação; e, iii) se os resultados podem ser usados em generalizações para toda a população quando se está testando a associação em amostras". Ainda de acordo com o autor, o coeficiente de associação $\mathrm{Q}_{\mathrm{xy}}$ apresenta dois postulados base: (1) deve ser igual a zero quando as variáveis $\mathrm{X}$ e Y forem independentes; e (2) deve ser de, no máximo, +1,00 no caso de associações positivas e de $-1,00$ no caso de associações negativas (Cervi, 2019, p. 40).

A fórmula para a aplicação do Q de Yule é a seguinte (Yule e Kendall, 1937):

\footnotetext{
${ }^{14}$ Ver também: Sexton e Decker (1992); Barro e Lee (2005).

${ }^{15}$ Os dados completos estão disponíveis no Apêndice 1.
} 


$$
Q_{x y}=\frac{(B \times C)-(A \times D)}{(B \times C)+(A \times D)}
$$

Onde, em uma tabela quádrupla usada para representar as possíveis combinações existentes entre as duas variáveis dicotômicas, temos:

TABELA 1 - INTERVALOS DE VALORES PARA COEFICIENTE Qxy

\begin{tabular}{|c|c|c|c|}
\cline { 2 - 4 } \multicolumn{1}{c|}{} & Não- $Y$ & $Y$ & Total \\
\hline$X$ & $A$ & $B$ & Marginal X \\
\hline Não- $X$ & $C$ & $D$ & Marginal Não-Y \\
\hline Total & Marginal Não-Y & Marginal Y & Total de Casos (N) \\
\hline
\end{tabular}

FONTE: Cervi (2019).

Partindo ainda dos postulados básicos do Coeficiente de Yule, Davis (1976) sugere a forma adequada de interpretar os valores de $\mathrm{Q}_{\mathrm{xy}}$, organizados por grau de intensidade conforme exposto na Tabela 2.

TABELA 2 - INTERVALOS DE VALORES PARA COEFICIE
\begin{tabular}{|c|c|}
\hline Valor de $Q_{x y}$ & Leitura \\
\hline$+0,7$ ou mais & Associação positiva muito forte; \\
\hline$+0,5$ a $+0,69$ & Associação positiva forte; \\
\hline$+0,3$ a $+0,49$ & Associação positiva moderada; \\
\hline$+0,1$ a $+0,29$ & Associação positiva baixa; \\
\hline$+0,01$ a $+0,09$ & Associação positiva desprezível; \\
\hline 0 & Associação inexistente; \\
\hline$-0,01$ a $-0,09$ & Associação negativa desprezível; \\
\hline$-0,1$ a $-0,29$ & Associação negativa baixa; \\
\hline$-0,3$ a $-0,49$ & Associação negativa moderada; \\
\hline$-0,5$ a $-0,69$ & Associação negativa forte; e \\
\hline$-0,7$ ou mais & Associação negativa muito forte. \\
\hline
\end{tabular}

FONTE: Davis (1976, p. 70).

Apresentado o método de análise que foi empregado, passa-se agora para a apresentação dos resultados básicos, onde foi calculado o Coeficiente de Associação de Yule para cada uma das quatro hipóteses testadas. 


\section{RESULTADOS}

Considerando que o presente artigo busca verificar a validade de quatro hipóteses distintas, foi feita a opção metodológica de testar cada uma dessas hipóteses individualmente ${ }^{16}$, buscando o Coeficiente de Associação de Yule referente a cada uma delas. Assim, sendo a primeira hipótese

$H_{1}$ : Um Estado é mais propenso a votar junto com EUA na AGNU quando seu chefe de Estado teve contato prévio com instituições de ensino norte-americanas.

Temos:

TABELA 3 - TABELA QUÁDRUPLA PARA A HIPÓTESE 1

\begin{tabular}{|c|c|c|c|}
\cline { 2 - 4 } \multicolumn{1}{c|}{} & Não- $Y$ & $Y$ & Total \\
\hline$X$ & 1.108 & 750 & $1.858(5,72 \%)$ \\
\hline Não- $X$ & 20.995 & 9.608 & $30.603(94,28 \%)$ \\
\hline Total & $22.103(68,09 \%)$ & $10.358(31,91 \%)$ & 32.461 \\
\hline
\end{tabular}

FONTE: Dados da pesquisa.

Onde:

$X=$ Votou com os EUA em determinada votação; e

$Y=O$ chefe de Estado daquele país, durante aquela votação, havia tido contato prévio com instituições de ensino norte-americanas.

Aplicando a fórmula do $\mathrm{Q}_{\mathrm{xy}}$ :

$$
Q_{x y}=\frac{(B \times C)-(A \times D)}{(B \times C)+(A \times D)}=\frac{(750 \times 20.995)-(1.108 \times 9.608)}{(750 \times 20.995)+(1.108 \times 9.608)}=\frac{5.100 .586}{26.391 .914}=+0,193
$$

Aplicando o mesmo teste nas demais hipóteses levantadas pela pesquisa, os resultados foram os seguintes:

$\mathrm{H}_{2}$ : Um Estado é mais propenso a votar junto com EUA na AGNU, em pautas consideradas importantes pelo governo norte-americano, quando seu chefe de Estado teve contato prévio com instituições de ensino norte-americanas.

\footnotetext{
${ }^{16} \mathrm{Em}$ todas as hipóteses o valor-p retornou resultados inferiores a 0,001.
} 
TABELA 4 - TABELA QUÁDRUPLA PARA A HIPÓTESE 2

\begin{tabular}{|c|c|c|c|}
\cline { 2 - 4 } \multicolumn{1}{c|}{} & Não- $Y$ & $Y$ & Total \\
\hline$X$ & 782 & 510 & $1.292(24,78 \%)$ \\
\hline Não- $X$ & 2.736 & 1.185 & $3.921(75,22 \%)$ \\
\hline Total & $3.518(67,49 \%)$ & $1.695(32,51 \%)$ & 5.213 \\
\hline
\end{tabular}

FONTE: Dados da pesquisa.

Onde:

$X=$ Votou com os EUA em determinada votação indicada como importante pelo governo norte-americano; $\mathrm{e}$

$Y=O$ chefe de Estado daquele país, durante aquela votação, havia tido contato prévio com instituições de ensino norte-americanas.

$$
Q_{x y}=\frac{(B \times C)-(A \times D)}{(B \times C)+(A \times D)}=\frac{(510 \times 2.736)-(782 \times 1.185)}{(510 \times 2.736)+(782 \times 1.185)}=\frac{468.690}{2.322 .030}=+0,202
$$

H.: Um Estado é mais propenso a votar junto com EUA na AGNU quando seu chefe de Estado teve contato prévio com instituições de ensino norte-americanas, ou de outros membros da OTAN.

TABELA 5 - TABELA QUÁDRUPLA PARA A HIPÓTESE 3

\begin{tabular}{|c|c|c|c|}
\cline { 2 - 4 } \multicolumn{1}{c|}{} & Não- $Y$ & $Y$ & Total \\
\hline$X$ & 751 & 1.107 & $1.858(5,72 \%)$ \\
\hline Não- $X$ & 13.992 & 16.611 & $30.603(94,28 \%)$ \\
\hline Total & $14.743(45,42 \%)$ & $17.718(54,58 \%)$ & 32.461 \\
\hline
\end{tabular}

FONTE: Dados da pesquisa.

Onde:

$X=$ Votou com os EUA em determinada votação;

$Y=O$ chefe de Estado daquele país, durante aquela votação, havia tido contato prévio com instituições de ensino norte-americanas ou de outros membros da OTAN.

$$
Q_{x y}=\frac{(B \times C)-(A \times D)}{(B \times C)+(A \times D)}=\frac{(1.107 \times 13.992)-(751 \times 16.611)}{(1.107 \times 13.992)+(751 \times 16.611)}=\frac{3.014 .283}{27.964 .005}=+0,108
$$


H4: Um Estado é mais propenso a votar junto com EUA na AGNU, em pautas consideradas importantes pelo governo norte-americano, quando seu chefe de Estado teve contato prévio com instituições de ensino norte-americanas, ou de outros membros da OTAN.

TABELA 6 - TABELA QUÁDRUPLA PARA A HIPÓTESE 4

\begin{tabular}{|c|c|c|c|}
\cline { 2 - 4 } \multicolumn{1}{c|}{} & Não- $Y$ & $Y$ & Total \\
\hline$X$ & 535 & 757 & $1.292(24,78 \%)$ \\
\hline Não- $X$ & 1.835 & 2.086 & $3.921(75,22 \%)$ \\
\hline Total & $2.370(45,46 \%)$ & $2.843(54,54 \%)$ & 5.213 \\
\hline
\end{tabular}

FONTE: Dados da pesquisa.

Onde:

$X=$ Votou com os EUA em determinada votação indicada como importante pelo governo norte-americano;

$Y=O$ chefe de Estado daquele país, durante aquela votação, havia tido contato prévio com instituições de ensino norte-americanas ou de outros membros da OTAN.

$$
Q_{x y}=\frac{(B \times C)-(A \times D)}{(B \times C)+(A \times D)}=\frac{(757 \times 1.835)-(535 \times 2.086)}{(757 \times 1.835)+(535 \times 2.086)}=\frac{273.085}{2.505 .105}=+0,109
$$

\section{DISCUSSÃO}

Retomando os postulados de Davis (1976) sobre a interpretação adequada para os valores de $\mathrm{Q}_{\mathrm{xy}}$, temos que todas as variações do teste retornaram resultados flutuando entre $+0,108$ e $+0,202$, caindo dentro do intervalo considerado pelo autor como "associações positivas baixas". A Tabela 7 resume os resultados do $\mathrm{Q}_{\mathrm{xy}}$ em todas as hipóteses testadas, diferenciando-as com base nas alterações das variáveis $\mathrm{X}$ e $\mathrm{Y}$.

TABELA 7 - RESUMO DOS COEFICIENTES Qxy NAS HIPÓTESES TESTADAS

\begin{tabular}{|c|c|c|}
\cline { 2 - 3 } \multicolumn{1}{c|}{} & $\begin{array}{c}\text { Votou com os EUA em } \\
\text { determinada votação }\end{array}$ & $\begin{array}{c}\text { Votou com os EUA em } \\
\text { determinada votação importante }\end{array}$ \\
\hline $\begin{array}{c}\text { Teve contato com instituições } \\
\text { de ensino estadunidenses }\end{array}$ & $+0,193\left(\mathrm{H}_{1}\right)$ & $+0,202\left(\mathrm{H}_{2}\right)$ \\
\hline $\begin{array}{c}\text { Teve contato com instituições } \\
\text { de ensino estadunidenses ou } \\
\text { de outros membros da OTAN }\end{array}$ & $+0,108\left(\mathrm{H}_{3}\right)$ & $+0,109\left(\mathrm{H}_{4}\right)$ \\
\hline
\end{tabular}

FONTE: Dados da pesquisa. 
A alteração na variável $X$, presente nas hipóteses 2 e 4, que considera apenas as votações indicadas como importantes pelo governo estadunidense, representou um aumento no coeficiente de associação de Yule se comparado às suas contrapartes nas hipóteses 1 e 3, que consideram as votações em sua totalidade. Tal aumento, contudo, foi marginal (de $+0,193$ para $+0,202$; e de $+0,108$ para $+0,109$ ), estando de acordo com estudos produzidos por outros autores que há anos indicam que o recorte exclusivo sobre as votações consideradas importantes pelos EUA não produz alterações significativas nos resultados das pesquisas (Kegley Jr. e McGowan, 1981; Wittkopf, 1973).

Por outro lado, ao alterar a variável Y, considerando também os chefes de Estado que tiveram contato com instituições de ensino de Estados aliados dos EUA (aqui recortados com base nos Estados membros da OTAN), os coeficientes $\mathrm{Q}_{\mathrm{xy}}$ sofreram uma queda digna de nota (de $+0,193$ para $+0,108$; e de $+0,202$ para +0,109), o que vai na direção de invalidar o argumento de que a socialização em Estados aliados também produziria resultados positivos para os EUA, reforçando a ideia de que a teoria pode ter algum fundamento, mas exclusivamente quando consideramos o contato com instituições estadunidenses.

\section{CONSIDERAÇÕES FINAIS}

Os resultados forneceram evidências que corroboram com a hipótese de que o contato prévio com instituições de ensino norte-americanas por parte de chefes de Estado produz uma postura mais positiva dessas nações em relação aos EUA. Apesar do impacto ser baixo, é relevante notar que o mesmo é produzido por políticas que custam relativamente muito pouco aos cofres públicos norteamericanos, apresentando uma excelente relação custo-benefício se comparada a outras políticas destinadas a ampliar a influência internacional dos EUA, como ajuda financeira internacional e gastos na indústria militar. Naturalmente, esses resultados são apenas o primeiro passo para verificar se a teoria se sustenta e mensurar os impactos reais que programas de intercâmbio destinados a futuros líderes exercem sobre o comportamento internacional dos Estados.

Alguns dos principais desafios encontrados no presente artigo dizem respeito as próprias limitações do desenho de pesquisa empregado. Apesar de apresentar a vantagem de ser estatisticamente testável em grande escala - razão pela qual este desenho de pesquisa foi escolhido -, este cai nos mesmos impasses já notados em pesquisas semelhantes, notavelmente: não ser capaz de mensurar a profundidade e extensão das interações sociais entre os estudantes e a população local 
(Atkinson, 2010); desconsiderar fatores como se o aluno recebeu uma bolsa ou pagou seus próprios estudos, além da possibilidade de ter tido experiências desapontadoras ou mesmo solitárias (Lomer, 2017); e não ser capaz de fatorar as chances da exposição prolongada a cultura e sociedade norteamericanas acabarem por destacar os aspectos negativos das mesmas, produzindo uma duradoura animosidade em relação ao país (Freyburg, 2015).

Em termos objetivos, os resultados apresentados comprovam que os Estados-membros do GRULAC, no período entre o fim da Guerra Fria e dezembro de 2017, foram mais propensos a votar junto com os EUA na AGNU quando governados por um chefe de Estado que havia tido contato com instituições de ensino norte-americanas no passado. Apesar da generalização ser especulável em termos teóricos, esses resultados carecem de confirmação para quaisquer casos que fujam ao que foi empiricamente testado. Assim, uma agenda de pesquisa futura nesse tema deve envolver a expansão do teste aqui executado, verificando sua validade em Estados que não fazem parte da região da América Latina e Caribe, além de procurar incluir as variáveis aqui apresentadas em modelos estatísticos mais completos voltados a entender os padrões de votação dos Estados na AGNU. 


\section{REFERÊNCIAS}

ANDERSEN, T. B.; HARR, T.; TARP, F. On US politics and IMF lending. European Economic Review, v. 50, n. 7, p. 1843-1862, 2006.

ATKINSON, C. Constructivist Implications of Material Power: Military Engagement and the Socialization of States 1972-2000. International Studies Quarterly, v. 50, p. 509-537, 2006.

Does Soft Power Matter? A Comparative Analysis of Student Exchange Programs 1980-2006.

Foreign Policy Analysis, v. 6, n. 1, p. 1-22, 2010.

BAILEY, M. A.; STREZHNEV, A.; VOETEN, E. United Nations General Assembly Voting Data. Harvard Database, v. 18, 2009.

Estimating Dynamic State Preferences from United Nations Voting Data. p. 1-27, 2015.

BARRO, R. J.; LEE, J.-W. IMF-Programs: who is chosen and what are the effects? Journal of Monetary Economics, n. 52, p. 1245-1269, 2005.

BECK, P. A.; JENNINGS, M. K. Family Traditions, Political Periods, and the Development of Partisan Orientations. Journal of Politics, v. 53, p. 742-763, 1991.

BELLIN, E. Reconsidering the Robustness of Authoritarianismin the Middle East. Comparative Politic, v. 44, n. 2, p. 127-149, 2012.

BENNIS, P. The United Nations and Palestine: partition and its aftermath-UN stance on Palestine's displacement by creation of Israel. Arab Studies Quarterly, v. 19, n. 3, p. 47-77, 1997.

BEYERS, J. Conceptual and methodological challenges in the study of European socialization. Journal of European Public Policy, v. 17, n. 6, p. 909-920, 2010.

CAMPBELL, A. K. UN Voting and Alliance Cohesion. Millennium: Journal of International Studies, v. 1, n. 2, p. 4-16, 1971.

CERVI, E. U. Manual de Métodos Quantitativos para iniciantes em Ciência Política - Volume 2. Curitiba: CPOP, 2019.

CHAYES, A.; CHAYES, A. H. The New Sovereignty: Compliance with International Regulatory Agreements. Cambridge: Harvard University Press, 1996.

CHECKEL, J. T. International institutions and socialization in Europe: Introduction and framework. International Organization, v. 59, n. 4, p. 801-826, 2005.

CIALDINI, R. Compliance Principles of Compliance Professionals: Psychologists of Necessity. In: ZANNA, M. P.; OLSON, J. M.; HERMAN, C. P. (Eds.). Social Influence: The Ontario Symposium, vol. 5. Hillsdale: Lawrence Erlbaum, 1987. p. 165-184.

COOPER, J.; BLACKMAN, S. F.; KELLER, K. T. The science of attitudes. New York: Routledge, 
2016.

COPE, J. A. International Military Education and Training: An Assessment. Washington, DC: National Defense University, Institute for National Strategic Studies, 1995.

DAVIS, J. A. Levantamento de Dados em Sociologia: uma análise estatística elementar. Rio de Janeiro: Zahar Editores, 1976.

DIMITROVA, A.; PRIDHAM, G. International actors and democracy promotion in central and eastern Europe: the integration model and its limits. Democratization, v. 11, n. 5, p. 91-112, 2004.

DREHER, A.; NUNNENKAMP, P.; THIELE, R. Does US aid buy UN general assembly votes? A disaggregated analysis. Public Choice, v. 136, n. 1-2, p. 139-164, 2008.

DREHER, A.; STURM, J. E. Do IMF and World Bank Influence Voting in the UN General Assembly? Thurgau Institute of Economics. Foreign Affairs, December 2004, p. 1-40, 2006.

FREYBURG, T. Transgovernmental networks as catalysts for democratic change? EU functional cooperation with arab authoritarian regimes and socialization of involved state officials into democratic governance. Democratization, v. 18, n. 4, p. 1001-1025, 2011.

- Transgovernmental Networks as an Apprenticeship in Democracy? Socialization into Democratic Governance through Cross-national Activities. International Studies Quarterly, v. 59, n. 1, p. 59-72, 2015.

GIFT, T.; KRCMARIC, D. Who Democratizes? Western-educated Leaders and Regime Transitions. Journal of Conflict Resolution, v. 61, n. 3, p. 671-701, 2017.

GREENHILL, B. The Company You Keep: International Socialization and the Diffusion of Human Rights Norms. International Studies Quarterly, v. 54, p. 127-145, 2010.

HOLLOWAY, S. Forty Years of United Nations General Assembly Voting. Canadian Journal of Political Science, v. 23, n. 02, p. 279, 2009.

JOHNSTON, A. I. Treating international institutions as social environments. International Studies Quarterly, v. 45, n. 4, p. 487-515, 2001.

KEGLEY JR., C. W.; HOOK, S. J. U.S. foreign aid and U.N. voting: did Reagan's linkage strategy buy defence or defiance? International Studies Quarterly, n. 35, p. 295-312, 1991.

KEGLEY JR., C. W.; MCGOWAN, P. J. The Political Economy of Foreign Policy. Beverly Hills: SAGE Publications, 1981.

KELLEY, J. International Actors on the Domestic Scene: Membership Conditionality and Socialization by International Institutions. International Organization, v. 58, n. 3, p. 425-457, 2004.

KIM, S. Y.; RUSSETT, B. The new politics of voting alignments in the United Nations General Assembly. International Organization, v. 50, n. 04, p. 629, 1996.

LOMER, S. Soft power as a policy rationale for international education in the UK: a critical analysis. 
Higher Education, v. 74, n. 4, p. 581-598, 2017.

MILLER, A. H. Promoting Democratic Values in Transitional Societies through Foreign Aid. Midwest Political Science Association Annual Meeting. Chicago: 2006

MORAVCSIK, A. Explaining International Human Rights Regimes: Liberal Theory and Western Europe. European Journal of International Relations, v. 1, p. 157-190, 1995.

NAFSA. In America's Interest: Welcoming International Students, 2003. Disponível em: <https://www.nafsa.org/sites/default/files/ektron/uploadedFiles/NAFSA_Home/Resource_Library_ Assets/Public_Policy/in_america_s_interest.pdf>. Acesso em: 4 mar. 2020.

NAPIER, R. W.; GERSHENFELD, M. K. Groups: Theory and Experience. 4th Ed. ed. Boston: Hough- ton Mifflin, 1987.

NISBETT, R. E.; COHEN, D. Culture of Honor: The Psychology of Violence in the South. Boulder: Westview Press, 1996.

NYE, J. S. Soft Power: The Means to Sucess in World Politics. Cambridge: Public Affairs, 2004.

. Public diplomacy and soft power. Annals of the American Academy of Political and Social Science, v. 616, n. 1, p. 94-109, 2008.

PERLOFF, R. M. The Dynamics of Persuasion - Communication and Attitudes in the 21st Century. 6th Editio ed. New York: Routledge, 2017.

PEVEHOUSE, J. C. Democracy from the Outside-In? International Organizations and Democratization. International Organization, v. 56, n. 3, p. 515-549, 2002a.

With a Little Help from My Friends? Regional Organizations and the Consolidation of Democracy. American Journal of Political Science, v. 46, n. 3, p. 611-626, 2002 b.

Democracy from Above - Regional Organizations and Democratization. New York: Cambridge University Press, 2005.

POTRAFKE, N. Does government ideology influence political alignment with the U.S.? An empirical analysis of voting in the UN General Assembly. Review of International Organizations, v. 4, p. 245-268, 2009.

POWELL, C. L. Statement on International Education Week 2001, 2001. Disponível em: <https://2001-2009.state.gov/secretary/former/powell/remarks/2001/4462.htm>. Acesso em: 4 mar. 2020.

RICHMOND, Y. Cultural Exchange and the Cold War: Raising the Iron Curtain. University Park: Pennsylvania State University Press, 2003.

RUBY, T. Z.; GIBLER, D. US professional military education and democratization abroad. European Journal of International Relations, v. 16, n. 3, p. 339-364, 2010.

SELLTIZ, C. et al. Attitudes and Social Relations of Foreign Students in the United States. Minneapolis: University of Minnesota Press, 1963. 
SEXTON, E. A.; DECKER, T. N. U.S. foreign aid: is it for friends, development or politics. The Journal of Social, Political and Economic Studies, n. 17, p. 303-315, 1992.

STRYKER, S.; STATHAM, A. Symbolic Interaction and Role Theory. In: LINDZEY, G.; ARONSON, E. (Eds.). The Handbook of Social Psychology. New York: Random House, 1985. p. 311-378.

SUSSKIND, L. Environmental Diplomacy: Negotiating More Effective Global Agreements. London: Oxford University Press, 1994.

THACKER, S. C. The high politics of IMF lending. World Politics, n. 52, p. 38-75, 1999.

TURNER, J. C. Rediscovering the Social Group. Oxford: Basil Blackwell, 1987.

UNITED STATES DEPARTMENT OF STATE. Voting Practices in the United Nations 2017 Report to Congress Submitted Pursuant to Public Laws 101-246 and 108-447. Disponível em: <https://www.state.gov/wp-content/uploads/2019/05/Voting-Practices-in-the-United-Nations2017.pdf>. Acesso em: 3 abr. 2020.

WANG, X.-L. Cultural Mediators or Marginal Persons? Geographical Review, v. 81, p. 292-303, 1991.

WATSON, J.; LIPPITT, R. Cross-Cultural Experience as a Source of Attitude Change. Journal of Conflict Resolution, v. 2, p. 61-66, 1958.

WILSON, E. C.; BONILLA, F. Evaluating Exchange of Persons Programs. The Public Opinion Quarterly, v. 19, n. 20-30, 1955.

WITTKOPF, E. R. Foreign Aid and United Nations Votes: A Comparative Study. American Political Science Review, v. 67, n. 03, p. 868-888, 1973.

WOJCIUK, A.; MICHAŁEK, M.; STORMOWSKA, M. Education as a source and tool of soft power in international relations. European Political Science, v. 14, n. 3, p. 298-317, 2015.

YOUNG, O. The Effectiveness of International Institutions: Hard Cases and Critical Variables. In: ROSENAU, J. N.; CZEMPIEL, E. O. (Eds.). Governance Without Government: Order and Change in World Politics. Cambridge: Cambridge University Press, 1992. p. 160-194.

YULE, G. U.; KENDALL, M. G. An introduction to the theory of statistics. London: Charles Griffin, 1937. 


\section{APÊNDICES}

APÊNDICE A - DADOS PROSOPOGÁFICOS DOS CHEFES DE ESTADO DOS PAÍSES MEMBROS DO GRULAC $(1991-2017)^{17}$

\begin{tabular}{|c|c|c|c|c|}
\hline Chefe de Estado & Período d & o mandato & Instituição de ensino & País \\
\hline \multicolumn{5}{|c|}{ Antigua and Barbuda (Parlamentarismo) } \\
\hline Gaston Browne & $13 / 06 / 2014$ & - & University of Salamanca & Espanha \\
\hline Baldwin Spencer & $24 / 08 / 2004$ & $13 / 06 / 2014$ & Ruskin College & Reino Unido \\
\hline Lester Bird & 09/03/1994 & $24 / 08 / 2004$ & University of Michigan & EUA \\
\hline Vere Bird & $01 / 11 / 1981$ & 09/03/1994 & - & - \\
\hline \multicolumn{5}{|c|}{ Argentina (Presidencialismo) } \\
\hline Mauricio Macri & $10 / 12 / 2015$ & $10 / 12 / 2019$ & Columbia University & EUA \\
\hline Cristina Fernández de Kirchner & $10 / 12 / 2007$ & $09 / 12 / 2015$ & National University of La Plata & Argentina \\
\hline Néstor Kirchner & $25 / 05 / 2003$ & $10 / 12 / 2007$ & National University of La Plata & Argentina \\
\hline Eduardo Duhalde & $02 / 01 / 2002$ & $25 / 05 / 2003$ & University of Buenos Aires & Argentina \\
\hline Adolfo Rodríguez Saá & $23 / 12 / 2001$ & $30 / 12 / 2001$ & University of Buenos Aires & Argentina \\
\hline Fernando de la Rúa & 10/12/1999 & $21 / 12 / 2001$ & National University of Córdoba & Argentina \\
\hline Carlos Menem & 08/07/1989 & $10 / 12 / 1999$ & National University of Córdoba & Argentina \\
\hline \multicolumn{5}{|c|}{ Bahamas (Parlamentarismo) } \\
\hline Hubert Minnis & $11 / 05 / 2017$ & - & University of Minnesota & EUA \\
\hline Perry Christie & $08 / 05 / 2012$ & $10 / 05 / 2017$ & University of Birmingham & Reino Unido \\
\hline Hubert Ingraham & $04 / 05 / 2007$ & $08 / 05 / 2012$ & Government High School Institute & Bahamas \\
\hline Perry Christie & 03/05/2002 & $04 / 05 / 2007$ & University of Birmingham & Reino Unido \\
\hline Hubert Ingraham & 21/08/1992 & $03 / 05 / 2002$ & Government High School Institute & Bahamas \\
\hline \multicolumn{5}{|c|}{ Barbados (Parlamentarismo) } \\
\hline Freundel Stuart & $23 / 10 / 2010$ & $25 / 05 / 2018$ & $\begin{array}{l}\text { University of the West Indies at } \\
\text { Cave Hill }\end{array}$ & Barbados \\
\hline David Thompson & $16 / 01 / 2008$ & $23 / 10 / 2010$ & Combermere School & Barbados \\
\hline Owen Seymour Arthur & 06/09/1994 & $15 / 01 / 2008$ & $\begin{array}{l}\text { University of the West Indies at } \\
\text { Cave Hill }\end{array}$ & Barbados \\
\hline Erskine Sandiford & 01/06/1987 & $06 / 09 / 1994$ & University of the West Indies & Jamaica \\
\hline \multicolumn{5}{|c|}{ Belize (Parlamentarismo) } \\
\hline Dean Oliver Barrow & 08/02/2008 & - & University of Miami & EUA \\
\hline Said Wilbert Musa & 28/08/1998 & $08 / 02 / 2008$ & University of Manchester & Reino Unido \\
\hline Manuel Esquivel & 03/07/1993 & $28 / 08 / 1998$ & Loyola University New Orleans & EUA \\
\hline George Cadle Price & 07/09/1989 & 03/07/1998 & $\begin{array}{l}\text { Augustine's Minor Seminary in } \\
\text { Mississippi }\end{array}$ & EUA \\
\hline \multicolumn{5}{|c|}{ Bolivia (Presidencialismo) } \\
\hline Evo Morales & $22 / 01 / 2006$ & $10 / 11 / 2019$ & - & - \\
\hline Eduardo Rodríguez Veltzé & $09 / 06 / 2005$ & $22 / 01 / 2006$ & Harvard University & EUA \\
\hline Carlos Mesa & $17 / 10 / 2003$ & $09 / 06 / 2005$ & Complutense University of Madrid & Espanha \\
\hline Gonzalo Sánchez de Lozada & $06 / 08 / 2002$ & $17 / 10 / 2003$ & University of Chicago & EUA \\
\hline
\end{tabular}

\footnotetext{
${ }^{17}$ Nos casos em que um chefe de Estado tenha frequentado mais de uma instituição de ensino superior ou militar no mesmo país, apenas a instituição no qual se graduou foi considerada. Nos casos em quem chefe de Estado tenha frequentado mais de uma instituição, sendo uma delas nos EUA ou de outros Estados da OTAN, foi considerada apenas a instituição de ensino frequentada nestes Estados, dando primazia as instituições de ensino dos EUA.
} 


\begin{tabular}{|c|c|c|c|c|}
\hline Jorge Quiroga & $07 / 08 / 2001$ & $06 / 08 / 2002$ & Texas A\&M University & EUA \\
\hline Hugo Banzer & 06/08/1997 & $06 / 08 / 2001$ & US Army School of the Americas & EUA \\
\hline Gonzalo Sánchez de Lozada & 06/08/1993 & 06/08/1997 & University of Chicago & EUA \\
\hline Jaime Paz Zamora & 06/08/1989 & $06 / 08 / 1993$ & Universidade Católica de Lovaina & Bélgica \\
\hline \multicolumn{5}{|c|}{ Brasil (Presidencialismo) } \\
\hline Michel Temer & $12 / 05 / 2016$ & $31 / 12 / 2018$ & USP & Brasil \\
\hline Dilma Rousseff & $01 / 01 / 2011$ & $12 / 05 / 2016$ & UFMG & Brasil \\
\hline Luiz Inácio Lula da Silva & $01 / 01 / 2003$ & $31 / 12 / 2010$ & - & - \\
\hline Fernando Henrique Cardoso & 01/01/1995 & $31 / 12 / 2002$ & University of California (professor) & EUA \\
\hline Itamar Franco & $02 / 10 / 1992$ & $31 / 12 / 1994$ & UFJF & Brasil \\
\hline Fernando Collor de Mello & $15 / 03 / 1990$ & 02/10/1992 & UNB & Brasil \\
\hline \multicolumn{5}{|c|}{ Chile (Presidencialismo) } \\
\hline Michelle Bachelet & $11 / 03 / 2014$ & $11 / 03 / 2018$ & Humboldt University of Berlin & Alemanha \\
\hline Sebastián Piñera & $11 / 03 / 2010$ & $11 / 03 / 2014$ & Harvard University & EUA \\
\hline Michelle Bachelet & $11 / 03 / 2006$ & $11 / 03 / 2010$ & Humboldt University of Berlin & Alemanha \\
\hline Ricardo Lagos & $11 / 03 / 2000$ & $11 / 03 / 2006$ & Duke University & EUA \\
\hline Eduardo Frei Ruiz-Tagle & 11/03/1994 & $11 / 03 / 2000$ & University of Chile & Chile \\
\hline Patricio Aylwin & $11 / 03 / 1990$ & $11 / 03 / 1994$ & University of Chile & Chile \\
\hline \multicolumn{5}{|c|}{ Colombia (Presidencialismo) } \\
\hline Juan Manuel Santos & $07 / 08 / 2010$ & $07 / 08 / 2018$ & University of Kansas & EUA \\
\hline Álvaro Uribe & $07 / 08 / 2002$ & $07 / 08 / 2010$ & University of Oxford & Reino Unido \\
\hline Andrés Pastrana Arango & $07 / 08 / 1998$ & $07 / 08 / 2002$ & Harvard University & EUA \\
\hline Ernesto Samper Pizano & $07 / 08 / 1994$ & $07 / 08 / 1998$ & Columbia University & EUA \\
\hline César Gaviria & 07/08/1990 & 07/08/1994 & University of the Andes & Colombia \\
\hline \multicolumn{5}{|c|}{ Costa Rica (Presidencialismo) } \\
\hline Luis Guillermo Solís & $08 / 05 / 2014$ & $08 / 05 / 2018$ & Tulane University & EUA \\
\hline Laura Chinchilla & $08 / 05 / 2010$ & $08 / 05 / 2014$ & Georgetown University & EUA \\
\hline Óscar Arias & $08 / 05 / 2006$ & $08 / 05 / 2010$ & Boston University & EUA \\
\hline Abel Pacheco de La Espriella & $08 / 05 / 2002$ & $08 / 05 / 2006$ & Louisiana State University & EUA \\
\hline Miguel Ángel Rodríguez & 08/05/1998 & $08 / 05 / 2002$ & University of California, Berkeley & EUA \\
\hline José María Figueres Olsen & $08 / 05 / 1994$ & $08 / 05 / 1998$ & United States Military Academy & EUA \\
\hline $\begin{array}{l}\text { Rafael Ángel Calderón } \\
\text { Fournier }\end{array}$ & 08/05/1990 & 08/05/1994 & University of Costa Rica. & Costa Rica \\
\hline \multicolumn{5}{|c|}{ Cuba (Rep. Socialista de partido único) } \\
\hline Raúl Castro & $19 / 04 / 2011$ & - & University of Havana & Cuba \\
\hline Fidel Castro & $03 / 10 / 1965$ & $19 / 04 / 2011$ & University of Havana & Cuba \\
\hline \multicolumn{5}{|c|}{ Dominica (Parlamentarismo) } \\
\hline Roosevelt Skerrit & 08/01/2004 & - & New Mexico State University & EUA \\
\hline Pierre Charles & $03 / 10 / 2000$ & $06 / 01 / 2004$ & St. Mary's Academy & Dominica \\
\hline Roosevelt Bernard Douglas & $03 / 02 / 2000$ & $01 / 10 / 2000$ & Ontario Agricultural College & Canada \\
\hline Edison Chenfil James & $14 / 06 / 1995$ & $03 / 02 / 2000$ & University of East London & Reino Unido \\
\hline Eugenia Charles & $21 / 07 / 1980$ & $14 / 06 / 1995$ & London School of Economics & Reino Unido \\
\hline \multicolumn{5}{|c|}{ Rep. Dominicana (Presidencialismo) } \\
\hline Danilo Medina & $16 / 08 / 2012$ & - & $\begin{array}{l}\text { Santo Domingo Institute of } \\
\text { Technology }\end{array}$ & $\begin{array}{l}\text { Rep. } \\
\text { Dominicana }\end{array}$ \\
\hline Leonel Fernández & $16 / 08 / 2004$ & $16 / 08 / 2012$ & $\begin{array}{l}\text { Autonomous University of Santo } \\
\text { Domingo }\end{array}$ & $\begin{array}{l}\text { Rep. } \\
\text { Dominicana }\end{array}$ \\
\hline
\end{tabular}




\begin{tabular}{|c|c|c|c|c|}
\hline Hipólito Mejía & $16 / 08 / 2000$ & $16 / 08 / 2004$ & North Carolina State University & EUA \\
\hline Leonel Fernández & 16/08/1996 & $16 / 08 / 2000$ & $\begin{array}{l}\text { Autonomous University of Santo } \\
\text { Domingo }\end{array}$ & $\begin{array}{l}\text { Rep. } \\
\text { Dominicana }\end{array}$ \\
\hline Joaquín Balaguer & $16 / 08 / 1986$ & 16/08/1996 & University of Paris & France \\
\hline \multicolumn{5}{|c|}{ Equador (Presidencialismo) } \\
\hline Lenín Moreno & $24 / 05 / 2017$ & - & Central University of Ecuador & Equador \\
\hline Rafael Correa & $15 / 01 / 2007$ & $24 / 05 / 2017$ & University of Illinois & EUA \\
\hline Alfredo Palacio & $20 / 04 / 2005$ & $15 / 01 / 2007$ & Case Western Reserve University & EUA \\
\hline Lucio Gutiérrez & $15 / 01 / 2003$ & $20 / 04 / 2005$ & Escuela Politécnica del Ejército & Equador \\
\hline Gustavo Noboa & $22 / 01 / 2000$ & $15 / 01 / 2003$ & University of Guayaquil & Equador \\
\hline Jamil Mahuad & 10/08/1998 & $21 / 01 / 2000$ & Harvard University & EUA \\
\hline Fabián Alarcón & $11 / 02 / 1997$ & $10 / 08 / 1998$ & $\begin{array}{l}\text { Pontificia Universidad Católica del } \\
\text { Ecuador }\end{array}$ & Equador \\
\hline Abdalá Bucaram & $10 / 08 / 1996$ & 06/02/1997 & University of Guayaquil & Equador \\
\hline Sixto Durán-Ballén & $10 / 08 / 1992$ & $10 / 08 / 1996$ & Columbia University & EUA \\
\hline \multicolumn{5}{|c|}{ El Salvador (Presidencialismo) } \\
\hline Salvador Sánchez Cerén & $01 / 06 / 2014$ & $01 / 06 / 2019$ & Escuela Alberto Masferrer & El Salvador \\
\hline Mauricio Funes & $01 / 06 / 2009$ & $01 / 06 / 2014$ & Central American University & El Salvador \\
\hline Elías Antonio Saca González & $01 / 06 / 2004$ & $01 / 06 / 2009$ & Central American University & El Salvador \\
\hline Francisco Flores Pérez & $01 / 06 / 1999$ & $01 / 06 / 2004$ & Amherst College & EUA \\
\hline Armando Calderón Sol & 01/06/1994 & 01/06/1999 & $\begin{array}{l}\text { Universidad Nacional de El } \\
\text { Salvador }\end{array}$ & El Salvador \\
\hline Alfredo Cristiani & $01 / 06 / 1989$ & $01 / 06 / 1994$ & Georgetown University & EUA \\
\hline \multicolumn{5}{|c|}{ Granada (Parlamentarismo) } \\
\hline Keith Mitchell & $20 / 02 / 2013$ & - & Howard University & EUA \\
\hline Tillman Thomas & 09/07/2008 & $20 / 02 / 2013$ & Fordham University & EUA \\
\hline Keith Mitchell & $22 / 06 / 1995$ & 09/07/2008 & Howard University & EUA \\
\hline George Ignatius Brizan & 01/02/1995 & $22 / 06 / 1995$ & University of Calgary & Canada \\
\hline Nicholas Brathwaite & $16 / 03 / 1990$ & $01 / 02 / 1995$ & - & - \\
\hline \multicolumn{5}{|c|}{ Guatemala (Presidencialismo) } \\
\hline Jimmy Morales & $14 / 01 / 2016$ & - & $\begin{array}{l}\text { Universidad de San Carlos de } \\
\text { Guatemala }\end{array}$ & Guatemala \\
\hline Alejandro Maldonado & 03/09/2015 & $14 / 01 / 2016$ & $\begin{array}{l}\text { Universidad de San Carlos de } \\
\text { Guatemala }\end{array}$ & Guatemala \\
\hline Otto Pérez Molina & $14 / 01 / 2012$ & $03 / 09 / 2015$ & US Army School of the Americas & EUA \\
\hline Álvaro Colom & $14 / 01 / 2008$ & $14 / 01 / 2012$ & $\begin{array}{l}\text { Universidad de San Carlos de } \\
\text { Guatemala }\end{array}$ & Guatemala \\
\hline Óscar Berger & $14 / 01 / 2004$ & $14 / 01 / 2008$ & Rafael Landívar University & Guatemala \\
\hline Alfonso Portillo & $14 / 01 / 2000$ & $14 / 01 / 2004$ & Autonomous University of Guerrero & Mexico \\
\hline Álvaro Arzú & 14/01/1996 & $14 / 01 / 2000$ & Rafael Landívar University & Guatemala \\
\hline Ramiro de León Carpio & $06 / 06 / 1993$ & 14/01/1996 & $\begin{array}{l}\text { Universidad de San Carlos de } \\
\text { Guatemala }\end{array}$ & Guatemala \\
\hline Jorge Serrano & 14/01/1991 & $01 / 06 / 1993$ & Stanford University & EUA \\
\hline \multicolumn{5}{|c|}{ Guiana (Presidencialismo) } \\
\hline David A. Granger & $16 / 05 / 2015$ & - & Mons Officer Cadet School & Reino Unido \\
\hline Donald Ramotar & $03 / 12 / 2011$ & $16 / 05 / 2015$ & University of Guyana & Guyana \\
\hline Bharrat Jagdeo & $11 / 08 / 1999$ & 03/12/2011 & $\begin{array}{l}\text { Peoples' Friendship University of } \\
\text { Russia }\end{array}$ & Russia \\
\hline Janet Jagan & 19/12/1997 & $11 / 08 / 1999$ & Northwestern University & EUA \\
\hline
\end{tabular}




\begin{tabular}{|c|c|c|c|c|}
\hline Sam Hinds & 06/03/1997 & $19 / 12 / 1997$ & University of New Brunswick & Canada \\
\hline Cheddi Berret Jagan & $09 / 01 / 1992$ & 06/03/1997 & Howard University & EUA \\
\hline \multicolumn{5}{|c|}{ Haiti (Semi-presidencialismo) } \\
\hline Jovenel Moïse & $07 / 02 / 2017$ & - & Quisqueya University & Haiti \\
\hline Jocelerme Privert & $14 / 02 / 2016$ & $07 / 02 / 2017$ & - & - \\
\hline Michel Martelly & $14 / 05 / 2011$ & $07 / 02 / 2016$ & Miami-Dade Community College & EUA \\
\hline René Préval & $14 / 05 / 2006$ & $14 / 05 / 2011$ & University of Leuve & Bélgica \\
\hline Boniface Alexandre & $29 / 02 / 2004$ & $14 / 05 / 2006$ & - & - \\
\hline Jean-Bertrand Aristide & $07 / 02 / 2001$ & $29 / 02 / 2004$ & Université d'État d'Haïti & Haiti \\
\hline René Préval & 07/02/1996 & $07 / 02 / 2001$ & University of Leuve & Bélgica \\
\hline Jean-Bertrand Aristide & $12 / 10 / 1994$ & $07 / 02 / 1996$ & Université d'État d'Haïti & Haiti \\
\hline Émile Jonassaint & $15 / 06 / 1993$ & $12 / 10 / 1994$ & - & - \\
\hline Marc Louis Bazin & $19 / 06 / 1992$ & 15/06/1993 & Solvay Institute & Bélgica \\
\hline \multicolumn{5}{|c|}{ Honduras (Presidencialismo) } \\
\hline Juan Orlando Hernández & $27 / 01 / 2014$ & - & State University of New York & EUA \\
\hline Porfirio Lobo Sosa & $27 / 01 / 2010$ & $27 / 01 / 2014$ & University of Miami & EUA \\
\hline Roberto Micheletti & 28/06/2009 & 27/01/2010 & - & - \\
\hline Manuel Zelaya & $27 / 01 / 2006$ & 28/06/2009 & $\begin{array}{l}\text { National Autonomous University of } \\
\text { Honduras }\end{array}$ & Honduras \\
\hline Ricardo Maduro & $27 / 01 / 2002$ & $27 / 01 / 2006$ & Lawrenceville School & EUA \\
\hline Carlos Flores & $27 / 01 / 1998$ & $27 / 01 / 2002$ & Louisiana State University & EUA \\
\hline Carlos Roberto Reina & 27/01/1994 & 27/01/1998 & Cambridge University & Reino Unido \\
\hline Rafael Callejas & $27 / 01 / 1990$ & 27/01/1994 & Mississippi State University & EUA \\
\hline \multicolumn{5}{|c|}{ Jamaica (Parlamentarismo) } \\
\hline Andrew Holness & 03/03/2016 & - & University of the West Indies & Jamaica \\
\hline Portia Simpson-Miller & $05 / 01 / 2012$ & 03/03/2016 & Union Institute \& University & EUA \\
\hline Andrew Holness & $23 / 10 / 2011$ & $05 / 01 / 2012$ & University of the West Indies & Jamaica \\
\hline Bruce Golding & $11 / 09 / 2007$ & $23 / 10 / 2011$ & University of the West Indies & Jamaica \\
\hline Portia Simpson-Miller & $30 / 03 / 2005$ & $11 / 09 / 2007$ & Union Institute \& University & EUA \\
\hline Percival Noel James Patterson & $30 / 03 / 1992$ & $30 / 03 / 2005$ & London School of Economics & Reino Unido \\
\hline \multicolumn{5}{|c|}{ México (Presidencialismo) } \\
\hline Enrique Peña Nieto & $01 / 12 / 2012$ & $30 / 11 / 2018$ & Panamerican University & Mexico \\
\hline Felipe Calderón & $01 / 12 / 2006$ & $30 / 11 / 2012$ & Harvard University & EUA \\
\hline Vicente Fox & $01 / 12 / 2000$ & $30 / 11 / 2006$ & Universidad Iberoamericana & Mexico \\
\hline Ernesto Zedillo & $01 / 12 / 1994$ & $30 / 11 / 2000$ & Yale University & EUA \\
\hline Carlos Salinas de Gortari & $01 / 12 / 1988$ & 30/11/1994 & Harvard University & EUA \\
\hline \multicolumn{5}{|c|}{ Nicaragua (Presidencialismo) } \\
\hline Daniel Ortega & $10 / 01 / 2007$ & - & Universidad Centroamericana & Nicaragua \\
\hline Enrique Bolaños Geyer & $10 / 01 / 2002$ & $10 / 01 / 2007$ & Saint Louis University & EUA \\
\hline José Arnoldo Alemán Lacayo & 10/01/1997 & $10 / 01 / 2002$ & $\begin{array}{l}\text { Universidad Nacional Autónoma de } \\
\text { Nicaragua }\end{array}$ & Nicaragua \\
\hline Violeta Chamorro & $25 / 04 / 1990$ & $10 / 01 / 1997$ & Blackstone College for Girls & EUA \\
\hline \multicolumn{5}{|c|}{ Panamá(Presidencialismo) } \\
\hline Juan Carlos Varela & $01 / 07 / 2014$ & $01 / 07 / 2019$ & Georgia Institute of Technology & EUA \\
\hline Ricardo Martinelli & 01/07/2009 & $01 / 07 / 2014$ & University of Arkansas, Fayetteville & EUA \\
\hline Martín Torrijos & $01 / 09 / 2004$ & 01/07/2009 & Texas A\&M University & EUA \\
\hline
\end{tabular}




\begin{tabular}{|c|c|c|c|c|}
\hline Mireya Moscoso & $01 / 09 / 1999$ & $01 / 09 / 2004$ & Miami Dade College & EUA \\
\hline $\begin{array}{l}\text { Ernesto Pérez-Balladares } \\
\text { González-Revilla }\end{array}$ & 01/09/1994 & 01/09/1999 & University of Notre Dame & EUA \\
\hline Guillermo Endara & $20 / 12 / 1989$ & $01 / 09 / 1994$ & New York University & EUA \\
\hline \multicolumn{5}{|c|}{ Paraguai (Presidencialismo) } \\
\hline Horacio Cartes & $15 / 08 / 2013$ & $15 / 08 / 2018$ & Spartan School of Aeronautics & EUA \\
\hline Federico Franco & $22 / 06 / 2012$ & $15 / 08 / 2013$ & National University of Asunción & Paraguai \\
\hline Fernando Lugo & $15 / 08 / 2008$ & $22 / 06 / 2012$ & Pontifical Gregorian University & Itália \\
\hline Nicanor Duarte & $15 / 08 / 2003$ & $15 / 08 / 2008$ & Catholic University of Asuncion & Paraguai \\
\hline Luis Ángel González Macchi & $29 / 03 / 1999$ & $15 / 08 / 2003$ & National University of Asunción & Paraguai \\
\hline Raúl Cubas Grau & 15/08/1998 & $29 / 03 / 1999$ & National University of Asunción & Paraguai \\
\hline Juan Carlos Wasmosy & $15 / 08 / 1993$ & $15 / 08 / 1998$ & National University of Asunción & Paraguai \\
\hline Andrés Rodríguez & 03/02/1989 & 15/08/1993 & Escuela Militar de Asunción & Paraguai \\
\hline \multicolumn{5}{|c|}{ Peru $($ Presidencialismo $)$} \\
\hline Pedro Pablo Kuczynski & $28 / 07 / 2016$ & $23 / 03 / 2018$ & Princeton University & EUA \\
\hline Ollanta Humala & $28 / 07 / 2011$ & $28 / 07 / 2016$ & Chorrillos Military School & Peru \\
\hline Alan García & $28 / 07 / 2006$ & $28 / 07 / 2011$ & Complutense University of Madrid & Espanha \\
\hline Alejandro Toledo & $28 / 07 / 2001$ & $28 / 07 / 2006$ & University of San Francisco & EUA \\
\hline Valentín Paniagua & $22 / 11 / 2000$ & 28/07/2001 & $\begin{array}{l}\text { National University of San Antonio } \\
\text { Abad in Cuzco }\end{array}$ & Peru \\
\hline Alberto Fujimori & 28/07/1990 & $22 / 11 / 2000$ & University of Wisconsin & EUA \\
\hline \multicolumn{5}{|c|}{ São Cristóvão e Névis (Parlamentarismo) } \\
\hline Timothy Harris & $18 / 02 / 2015$ & - & McGill University & Canada \\
\hline Denzil Llewellyn Douglas & 06/07/1995 & $18 / 02 / 2015$ & $\begin{array}{l}\text { University of the West Indies at } \\
\text { Cave Hill }\end{array}$ & Barbados \\
\hline Kennedy Alphonse Simmonds & $19 / 09 / 1983$ & 06/07/1995 & - & - \\
\hline \multicolumn{5}{|c|}{ Santa Lúcia (Parlamentarismo) } \\
\hline Allen Chastanet & 07/06/2016 & - & American University & EUA \\
\hline Kenny Anthony & $30 / 11 / 2011$ & $07 / 06 / 2016$ & University of Birmingham & Reino Unido \\
\hline Stephenson King & 01/05/2007 & $30 / 11 / 2011$ & - & - \\
\hline John Compton & $11 / 12 / 2006$ & $01 / 05 / 2007$ & University College Wales & Reino Unido \\
\hline Kenny Anthony & $24 / 05 / 1997$ & $11 / 12 / 2006$ & University of Birmingham & Reino Unido \\
\hline Vaughan Lewis & $02 / 04 / 1996$ & $24 / 05 / 1997$ & - & - \\
\hline John Compton & 03/05/1982 & $02 / 04 / 1996$ & University College Wales & Reino Unido \\
\hline \multicolumn{5}{|c|}{ São Vicente e Granadinas (Parlamentarismo) } \\
\hline Ralph Gonsalves & $28 / 03 / 2001$ & - & University of Manchester & Reino Unido \\
\hline Arnhim Ulric Eustace & $27 / 10 / 2000$ & $28 / 03 / 2001$ & Sir George William University & Canada \\
\hline James Fitz-Allen Mitchell & $30 / 07 / 1984$ & $27 / 10 / 2000$ & University of British Columbia & Canada \\
\hline \multicolumn{5}{|c|}{ Suriname (Presidencialismo) } \\
\hline Dési Bouterse & $12 / 08 / 2010$ & - & Koninklijke Militaire School & Bélgica \\
\hline Ronald Venetiaan & $12 / 08 / 2000$ & $12 / 08 / 2010$ & Leiden University & Bélgica \\
\hline Jules Wijdenbosch & $15 / 09 / 1996$ & $12 / 08 / 2000$ & University of Amsterdam & Países Baixos \\
\hline Ronald Venetiaan & $16 / 09 / 1991$ & $15 / 09 / 1996$ & Leiden University & Bélgica \\
\hline \multicolumn{5}{|c|}{ Trinidad e Tobago (Parlamentarismo) } \\
\hline Keith Christopher Rowley & 09/09/2015 & - & University of the West Indies & Barbados \\
\hline Kamla Persad-Bissessar & $26 / 05 / 2010$ & $09 / 09 / 2015$ & University of the West Indies & Barbados \\
\hline Patrick Manning & $24 / 12 / 2001$ & $26 / 05 / 2010$ & University of the West Indies & Barbados \\
\hline
\end{tabular}


I Seminário Discente de Ciência Política (SDCP)

Programa de Pós-Graduação em Ciência Política (PPGCP)

Universidade Federal do Paraná (UFPR)

\begin{tabular}{|c|c|c|c|c|}
\hline Basdeo Panday & 09/11/1995 & $24 / 12 / 2001$ & University of London & Reino Unido \\
\hline Patrick Manning & $17 / 12 / 1991$ & 09/11/1995 & University of the West Indies & Barbados \\
\hline \multicolumn{5}{|c|}{ Uruguai (Presidencialismo) } \\
\hline Tabaré Vázquez & $01 / 03 / 2015$ & - & Gustave Roussy Institute & France \\
\hline José Mujica & 01/03/2010 & $01 / 03 / 2015$ & - & - \\
\hline Tabaré Vázquez & $01 / 03 / 2005$ & $01 / 03 / 2010$ & Gustave Roussy Institute & France \\
\hline Jorge Batlle & $01 / 03 / 2000$ & $01 / 03 / 2005$ & University of the Republic & Uruguai \\
\hline Julio María Sanguinetti & $01 / 03 / 1995$ & $01 / 03 / 2000$ & University of the Republic & Uruguai \\
\hline Luis Alberto Lacalle & $01 / 03 / 1990$ & $01 / 03 / 1995$ & University of the Republic & Uruguai \\
\hline \multicolumn{5}{|c|}{ Venezuela (Presidencialismo) } \\
\hline Nicolás Maduro & 05/03/2013 & - & - & - \\
\hline Hugo Chávez & 02/02/1999 & $05 / 03 / 2013$ & $\begin{array}{l}\text { Venezuelan Academy of Military } \\
\text { Sciences }\end{array}$ & Venezuela \\
\hline Rafael Caldera & 02/02/1994 & 02/02/1999 & Central University of Venezuela & Venezuela \\
\hline Ramón José Velásquez & 05/06/1993 & 02/02/1994 & Central University of Venezuela & Venezuela \\
\hline Carlos Andrés Pérez & 02/02/1989 & 21/05/1993 & Central University of Venezuela & Venezuela \\
\hline
\end{tabular}

Fonte: dados da pesquisa. 